\title{
ANALYTIC HIERARCHY PROCESS TO ASSESS TECHNOLOGICAL SYSTEM IN WATER TREATMENT PLANTS
}

\author{
Claudio Macuada *, Astrid Oddershede \\ Industrial Engineering Department, \\ University of Santiago of Chile, \\ Santiago, Chile. \\ E-mail: claudio.macuada@usach.cl, \\ E-mail: astrid.oddershede@usach.cl
}

\begin{abstract}
This paper proposes a multi-criteria approach to assess current technology systems in water treatment (WT) plants and water waste treatment (WWT) plants to find out the main implications on the quality of service. Presently, the plant production process and efficiency improvement of WWT plants has become a challenge for WWT plants. The need of cost-efficient and reliable treatment processes has significantly increased so as to meet the level of environmental regulations and national goals. A case study has been carry out in a main WWT company in Chile with the purpose to identify priority plants that must improve its technological standard. We have considered five technological systems that are indispensable in the process. With empirical data, expert opinions and combining scoring method with the Analytic Hierarchy Process (AHP) it was possible to obtain a plant ranking for the problem in study.
\end{abstract}

Keywords: MCDM, AHP, WWT technological systems. 


\section{Introduction}

Nowadays, there is a strong interest in increasing the efficiency of water treatment processes and production at national and international level. The requirements related to WT have been intensified and must comply with national environmental standards and objectives. At present, the industry is adopting new technologies for its processes and there is confidence that this is a way to improve their function. In the Netherlands, (Euronews | SCI-TECH | hi-tech, 2012) Dutch universities and public / private sector as part of a project have developed innovative technologies for WWT, improving the capacity of its plants, operating with higher flow and satisfying the needs of clear water. Thus, the water company under study has considered essential their plants must operate with the most adequate technological system. Therefore, it is necessary to determine the current level of technological system of WT and WWT plants and thus generate actions to make improvements in those plants showing lower quality of service.

In the analysis a multiplicity of factors and objectives are perceived, plants differ in size, production capacity and functionality. The implementation of technology competes also with available funds that may influence the evaluation. In this context the analytic hierarchy process is suitable to deal with conflicting goals. The study is divided into two main phases: the first phase is devoted to examine the existing technology and its degree of importance for plant performance and the significant attributes and criteria that mostly impact the selection of a technology system for WT and WWT plants are to be identified. In the second phase a hierarchical structure model is constructed aligning the work with a team of experts (plant managers, project engineers and members of senior management). This team was made up of twelve people who assessed all plants transversely. This equipment was subsequently commissioned to assess the current status of plants under the technological criteria to weigh the AHP evaluation result. The next section provides a brief literature review. Section 3 presents the objective of the study, The methodology is indicated in section while some results of the case study are presented in section 5, the limitations on section 6 and finally some of the conclusions are indicated on section 7 .

\section{Literature Review}

In regard to previous related work, we mention the research of Macuada et al., (2013) where an AHP decision model is developed for automating WT plants. The study provided a basis for setting priorities and decision making (DM) to incorporate automated system to WT and WWT plants. Karimi et al., (2011) used the fuzzy method and one AHP extension to select a process for WT in Iran; Perez, Oddershede \& Mena (2010), explored the feasibility to set up an appropriate domestic WT technology system in a Chilean isolated city after a volcanic eruption, Anagnostopoulos, Gratziou \&Vavatsikos (2005) used AHP to evaluate various scenarios for a water treatment plant in Greece; Nakhaei \& Taheriyoun (2005), considered various technologies for the application of wastewater treatment process through fuzzy AHP (FAHP) and gray relational analysis(GRA). In a similar attempt, the purpose of this study is to develop an AHP model for evaluating the current level of technological system of WT and WWT plants to produce improvements in those plants showing lower quality of service.

\section{Hypotheses/Objectives}

The context of the problem addressed comprises WT and WWT plants from a main company of the Metropolitan Region. For drinking water production, the company keeps 
two large capacity treatment plants and twelve smaller facilities where raw water is processed. Moreover, the company also processes the waste water generated in their concession areas, using modern WWT plants allows its return to the receptors in optimal conditions for agricultural irrigation, in conformity with standards legislation. In this regard, the company has two major treatment plants, plus twelve other smaller plants located in rural towns. These facilities allow achieving wastewater decontamination coverage around $100 \%$.

The number of WT plants for drinking water depends on the demand estimation to ensure the quantity, quality and continuity of this service which is vital for the population. The Water Company is interested in recognizing WT and WWT plants that urgently needs to improve their technology system to meet standards. In addition to these requests, the implementation will involve investment, operating and maintenance costs, hence the objective is to determine the priority plants to endow with new technology and to consider its cost benefit analysis.

This study will allow obtaining knowledge about features and weaknesses of current technological systems, also identifying deficiencies in WT and WWT plants

\section{Research Design/Methodology}

The initial approach starts with i) Generating a rating scale for each technology system (Data Capture, Communications, Data Control, Server and Instrumentation) that comprise the attributes for each plant, and ii) Designing the hierarchical structure for plants technological systems, where the critical categories for each level and its relationships are learned.

The hierarchical structure to prescribe the technological level of each wastewater treatment and drinking water plant is designed with two levels. In Figure 1, the basic simplified hierarchical structure is displayed, being a representation of the analysis where:

Level 0: Indicates the target, the main objective: "Remote Technology System

Level 1: Includes technology systems and / or alternative attributes that will help identify the most critical factors are technological plants.

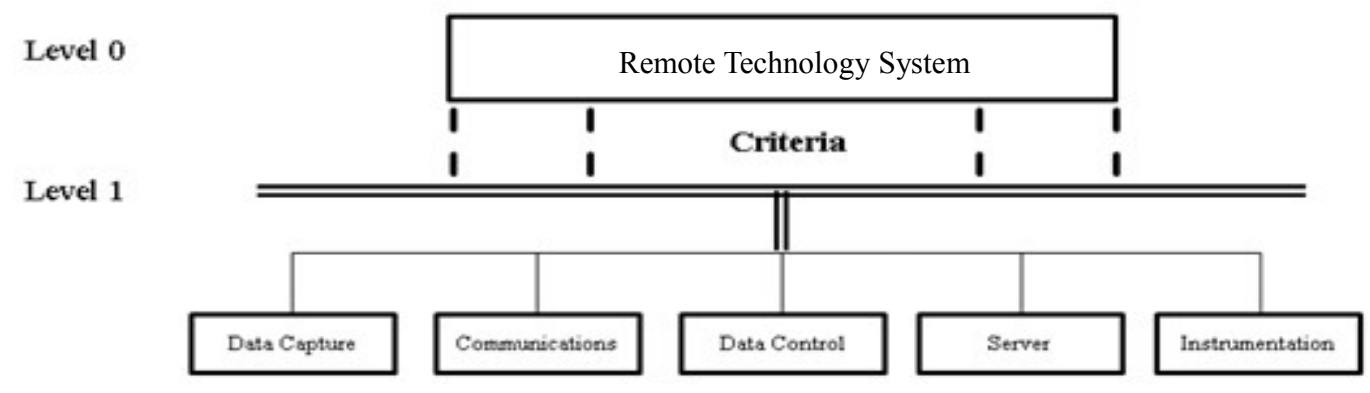

Figure 1 Hierarchical Basic Structure 
The decision factors are based on a benchmarking with similar size systems companies working 24 hours shifts, all linked to the treatment of industrial waste liquid. At this point, it was possible to be aware of certain existing taboos regarding to plants with a lower technological standard. A numerical score is obtained by the expert panel by reference to a table in order to measure current state of each plant. Then the outcome is included for AHP assessment of the most critical technological criteria for the final ranking and determine which plants are candidates for investment to increase their level of reliability.

\section{Data/Model Analysis}

Priority results indicated that there are three attributes that have equal relative weight: Instrumentation, Communications and Data Capture with $25 \%$, this is understood as they are the basic requirements to develop activities for the water treatment and comply with current legislation.

The results are consistent with the level of concern to the organization, to have plants that operate with high reliability and ensure that the plants are 24 hours online and meet the different requirements. Moreover, this result is directly related to the year of commissioning of the plants, because the study shows that there must be investment in plants that have many years in service and have not been updated technologically.

In this sense, the candidate plants are those with the lowest weighting in terms of the indispensable technology provision and functionality since there is less risk of generating conflict to the community in case of operational failure.

From this, it is interesting to view the different degree of importance given at the discretion of relevance to the company about what plants need to prioritize improving the technological standard. This result could be applied by people who make decisions for the distribution of resources, example is the investment to automate the operation versus having personnel operating plants or prevent failure requiring larger investments that can be made in today.

\section{Limitations}

The proposed model considers the technological systems with the same importance for plant production and water treatment. This was decided by the expert panel because the company contractor under study does not distinguish in terms of prices and guarantees, in line with current realities. You may however, consider this as separate analysis, in order to determine whether the relevant technological criteria are different depending on the type of treatment, in this case the production and depuration.

Regarding the financial aspects, this study have considered cost/benefit analysis for the selected plants in order to have notions about the economic aspects related to implementation

\section{Conclusions}

In this paper we have presented a real problem of resources prioritization that has not been done before in the company. The use of AHP methodology helped to inspect the actual technology provision of production $\&$ treatment water plants, and to determine the 
plants requiring achieving a technological standard. Consequently, the implementation will contribute to the improvement of the quality of service.

Given the existence of different decision variables to distinguish what plants would be candidates for technological improvements; the development of a decisional model using AHP was advantageous because a deep understanding of the problem situation was obtained. The expert panel participants valued the fact to know the plants present state, and through the process, the plants that need improvement.

The resulting prioritization indicates that investments are oriented to those plants with lower overall ranking; they indicate having less technological support reflected in a lower quality performance.

The cost/benefit analysis gives an idea in economic terms regarding the implementation of the chosen plants.

The results of this study can be seen in companies with industrial operation requiring treatments and need to assess their technological level, seeking to improve the operation, to provide better quality of service to the community.

\section{Key References}

Anagnostopoulos, K., Gratziou, M. and Vavatsikos, A. (2005). Evaluation of wastewater facilities scenarios with the use of the AHP multicriteria method. 9th International Conference on Environmental Science and Technology, Rhodes Island, Grecia.

Karimi, A., Mehrdadi, N., Hashemian, S., Nabi Bidhendi, G., \& Tavakkoli Moghaddam, R. (2011). Selection of wastewater treatment process based on the analytical hierarchy process and fuzzy analytical hierarchy process methods. International Journal of Environment Science and Technology, Vol. 8, No. 2, pp. 267-280.

Macuada, C., Alarcón, R., \& Oddershede, A. (2013). Multi-criteria assessment to automate water treatment plants using the Analytical Hierarchy Process. The 12th International Symposium on the Analytic Hierarchy Process, Kuala Lumpur, Malaysia.

Nakhaei, M. \& Taheriyoun, M. (2012). Evaluation of Wastewater Treatment Technologies Applying Fuzzy Analytic Hierarchy Process and Gray Relational Analysis. International Conference on Chemical, Civil and Environment engineering (ICCEE'2012), Dubai, UAE

Pérez, A., Oddershede, A., y Mena, M. (2013). Wastewater treatment system selection using the Analytical Hierarchy Process. The Operations Research Society Annual Conference OR52, Egham, London, UK.

Saaty T. L. (1997). Toma de Decisiones Para Líderes. El proceso jerárquico analítico. La toma de decisiones en un mundo complejo. RWS Publications, USA. 